Supplement of Biogeosciences, 17, 3471-3486, 2020

https://doi.org/10.5194/bg-17-3471-2020-supplement

(C) Author(s) 2020. This work is distributed under

the Creative Commons Attribution 4.0 License.

(c) (1)

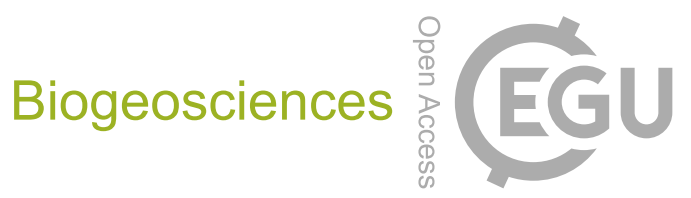

Supplement of

\title{
On giant shoulders: how a seamount affects the microbial community composition of seawater and sponges
}

Kathrin Busch et al.

Correspondence to: Ute Hentschel (uhentschel@geomar.de)

The copyright of individual parts of the supplement might differ from the CC BY 4.0 License. 


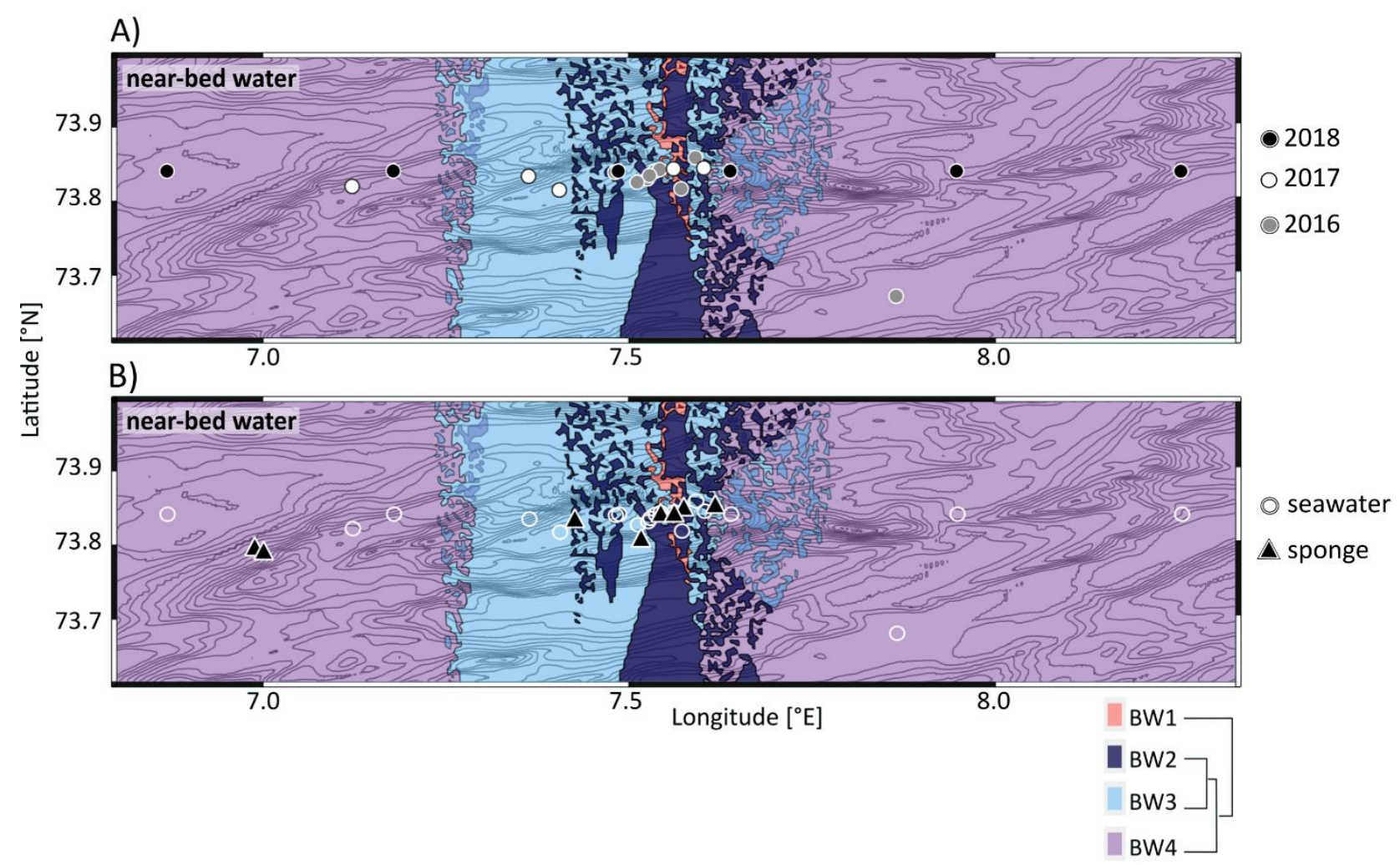

Supplementary Figure S1 Near-bed water microbial community structure across Schulz Bank in combination with either (A) information on sampling years of each seawater sampling station or (B) information on sampling locations of sponge samples (in relation to seawater sampling locations) across Schulz Bank. Contour lines in all three subplots represent the underlying topography. Colors in all subplots represent clusters based on weighted UniFrac distances, where colored dots indicate stations with in situ sampling and filled areas represent extrapolations based on machine learning. The degree of cluster similarity can be deduced from the dendrogram at the bottom of the plot. A) Different sampling years of the 19 CTD stations are indicated by colors of the dots. Samples from the 2018 cruise have a black color, samples from the 2017 cruise have a white color, and samples from the 2016 cruise have a grey color. B) Sponge sampling locations are marked by black triangles, while seawater sampling stations are indicated by white circles. 


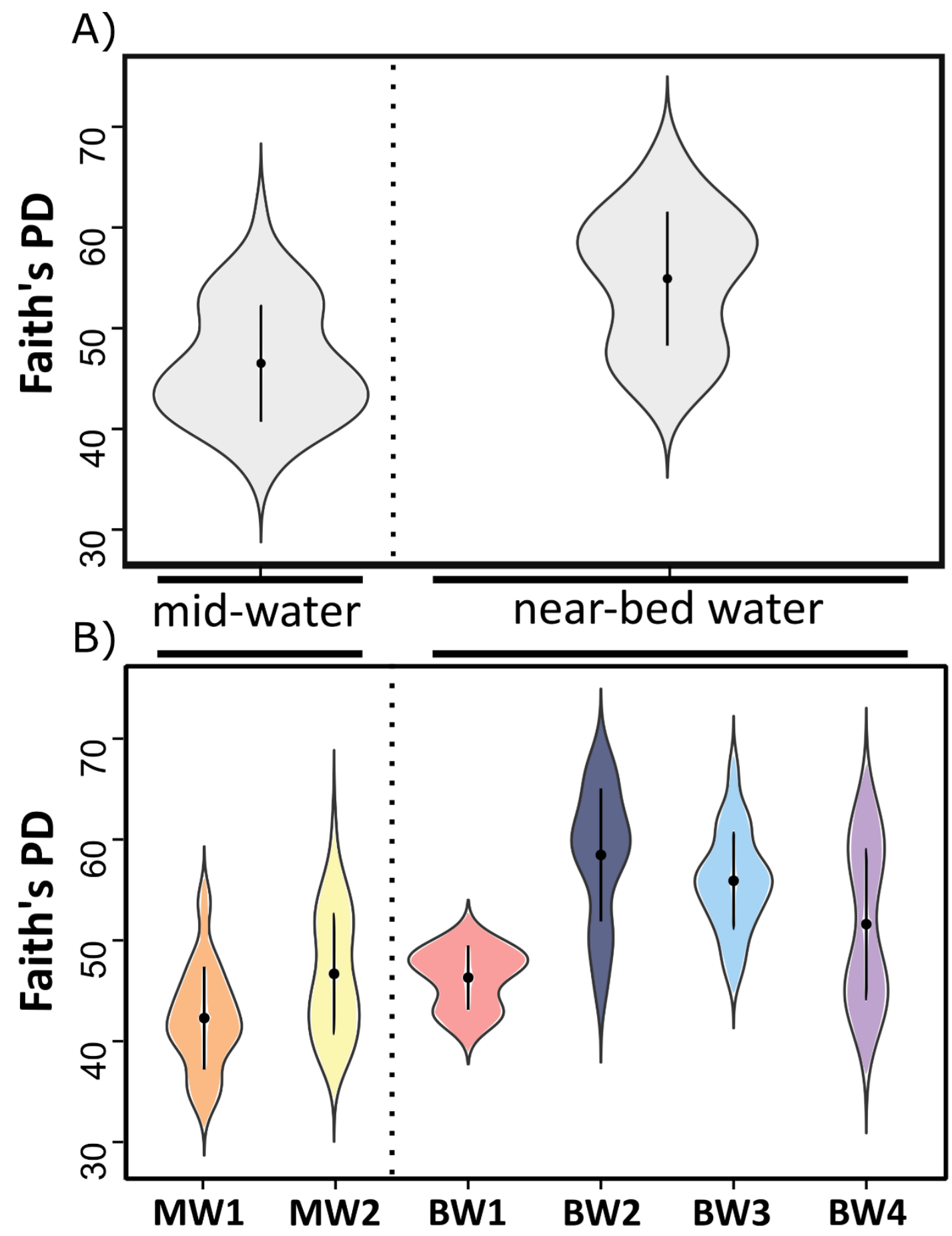

Supplementary Figure S2 A) Overall richness of pooled mid-water microbial communities and pooled near-bed water microbial communities. Faith Phylogenetic Diversity (Faith's PD) is plotted as alpha-diversity measure. B) Richness of seawater microbial communities for the mid-water and near-bed water samples, across the determined clusters. Mid-water clusters are coloured in orange (MW1) and yellow (MW2), while near-bed water clusters are marked in red (BW1), dark blue (BW2), light blue (BW3), and purple (BW4). 

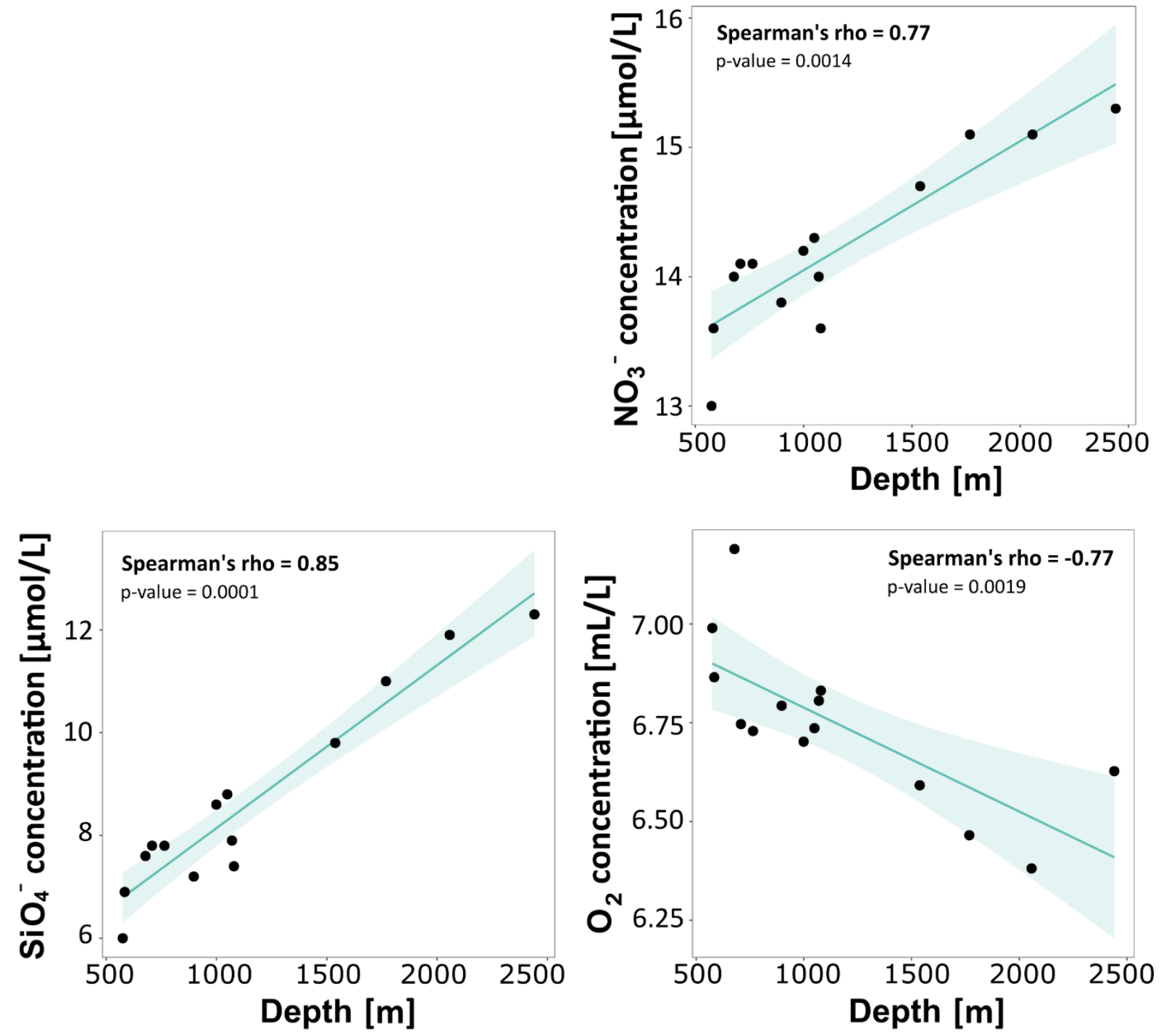

Supplementary Figure S3 Spearman's rank correlations calculated between depth and the three biogeochemical parameters which differed significantly (ANOVA, $\alpha=0.05$ ) across the determined near-bed water clusters. 
Supplementary Table S1 Overview of pairwise-comparisons (PERMANOVAs) across pelagic microbial micro-habitats based on weighted UniFrac matrices of microbial communities for both, seawater and the three sponge species. All possible pairwise combinations were tested. L. complicata and S. rosea were not present in all near-bed water clusters. Four biological replicates per near-bed water cluster were tested for every sponge species.

\begin{tabular}{|c|c|c|c|c|}
\hline Group 1 & Group 2 & pseudo-F & p-value & \\
\hline MW1 & MW2 & 22.49 & 0.001 & $*$ \\
\hline BW1 & BW2 & 27.7 & 0.004 & $*$ \\
\hline BW1 & BW3 & 11.79 & 0.002 & $*$ \\
\hline BW1 & BW4 & 15.07 & 0.003 & $*$ \\
\hline BW2 & BW3 & 8.52 & 0.001 & $*$ \\
\hline BW2 & BW4 & 15.56 & 0.001 & $*$ \\
\hline BW3 & BW4 & 10.98 & 0.001 & $*$ \\
\hline G. hentscheli BW1 & G. hentscheli BW2 & 5.77 & 0.032 & $*$ \\
\hline G. hentscheli BW1 & G. hentscheli BW3 & 8.07 & 0.028 & $*$ \\
\hline G. hentscheli BW1 & G. hentscheli BW4 & 16.71 & 0.030 & $*$ \\
\hline G. hentscheli BW2 & G. hentscheli BW3 & 4.57 & 0.039 & $*$ \\
\hline G. hentscheli BW2 & G. hentscheli BW4 & 14.41 & 0.035 & $*$ \\
\hline G. hentscheli BW3 & G. hentscheli BW4 & 5.57 & 0.028 & $*$ \\
\hline L. complicata BW1 & L. complicata BW2 & 35.93 & 0.034 & $*$ \\
\hline S. rosea BW1 & S. rosea BW2 & 1.88 & 0.035 & $*$ \\
\hline S. rosea BW1 & S. rosea BW3 & 1.71 & 0.174 & \\
\hline S. rosea BW2 & S. rosea BW3 & 1.18 & 0.36 & \\
\hline all MW & all BW & 46.72 & 0.001 & $*$ \\
\hline
\end{tabular}


Supplementary Table S2 Averages and standard errors of biogeochemical parameters for each mid-water and near-bed water cluster.

\begin{tabular}{|c|c|c|c|c|c|c|c|c|c|}
\hline & $\begin{array}{c}\text { SPM } \\
{\left[\mathrm{mg} \mathrm{L}^{-1}\right]}\end{array}$ & $\begin{array}{c}\text { DIC } \\
{\left[\mu \mathrm{mol} \mathrm{L}{ }^{-1}\right]}\end{array}$ & $\begin{array}{c}\mathrm{SiO}_{4}^{-} \\
{\left[\mu \mathrm{mol} \mathrm{L} \mathbf{L}^{-1}\right]}\end{array}$ & $\begin{array}{c}\mathrm{PO}_{4}{ }^{3-} \\
{\left[\mu \mathrm{mol} \mathrm{L} L^{-1}\right]}\end{array}$ & $\begin{array}{c}\mathrm{NH}_{4}^{+} \\
{\left[\mu \mathrm{mol} \mathrm{L}^{-1}\right]}\end{array}$ & $\begin{array}{c}\mathrm{NO}_{3}^{-} \\
{\left[\mu \mathrm{mol} \mathrm{L} \mathbf{L}^{-1}\right]}\end{array}$ & $\begin{array}{c}\mathrm{NO}_{2}^{-} \\
{\left[\mu \mathrm{mol} \mathrm{L} \mathbf{L}^{-1}\right]}\end{array}$ & $\begin{array}{c}\mathbf{O}_{2} \\
{\left[\mathbf{m L ~ L ~ L}^{-1}\right]}\end{array}$ & $\begin{array}{c}\text { Depth } \\
{[\mathrm{m}]}\end{array}$ \\
\hline MW1 & NA & $2246.00 \pm 6.99$ & $5.72 \pm 0.10$ & $0.88 \pm 0.02$ & NA & $12.84 \pm 0.08$ & $0.02 \pm 0.02$ & $6.90 \pm 0.04$ & $400 \pm 0$ \\
\hline MW2 & $2.22 \pm 1.39$ & $2292.14 \pm 53.64$ & $5.63 \pm 0.08$ & $0.84 \pm 0.01$ & $0.11 \pm 0.03$ & $13.08 \pm 0.11$ & $0.02 \pm 0.01$ & $6.75 \pm 0.03$ & $400 \pm 0$ \\
\hline $\begin{array}{c}\begin{array}{c}\text { significance } \\
\text { mid-water }\end{array} \\
\end{array}$ & & & & & & & & $*$ & \\
\hline BW1 & $0.49 \pm \mathrm{NA}$ & NA & 6.00 & 0.90 & 0.10 & 13.00 & 0.00 & $6.99 \pm \mathrm{NA}$ & 575 \\
\hline BW2 & NA & $2265.67 \pm 7.69$ & $8.40 \pm 0.31$ & $0.97 \pm 0.03$ & NA & $14.20 \pm 0.06$ & $0.00 \pm 0.00$ & $6.71 \pm 0.02$ & $919 \pm 106$ \\
\hline BW3 & $1.87 \pm 1.08$ & $2260.40 \pm 12.35$ & $7.87 \pm 0.42$ & $0.95 \pm 0.02$ & $0.17 \pm 0.03$ & $14.03 \pm 0.15$ & $0.03 \pm 0.02$ & $6.84 \pm 0.07$ & $922 \pm 142$ \\
\hline BW4 & $0.95 \pm 0.33$ & 2248.00 & $10.65 \pm 1.42$ & $0.95 \pm 0.03$ & $0.10 \pm 0.00$ & $14.78 \pm 0.52$ & $0.05 \pm 0.03$ & $6.48 \pm 0.10$ & $1836 \pm 376$ \\
\hline $\begin{array}{c}\text { significance } \\
\text { near-bed water }\end{array}$ & & & $*$ & & & $*$ & & $*$ & $*$ \\
\hline
\end{tabular}

